\title{
Mathematical Modeling and Analysis of Khat-Chewing Dynamics
}

\author{
Kahsay Godifey Wubneh, Fitsum Mulaw Desta, and Hafte Amsalu Kahsay (D) \\ Wollo University, College of Natural Science, Department of Mathematics, Dessie, Ethiopia \\ Correspondence should be addressed to Hafte Amsalu Kahsay; yohanahafte@gmail.com
}

Received 18 November 2020; Revised 21 January 2021; Accepted 29 January 2021; Published 13 February 2021

Academic Editor: Basil Papadopoulos

Copyright (c) 2021 Kahsay Godifey Wubneh et al. This is an open access article distributed under the Creative Commons Attribution License, which permits unrestricted use, distribution, and reproduction in any medium, provided the original work is properly cited.

\begin{abstract}
Khat is a green leaf and greenish plant where its branches and leaves are chewed to discharge liquid having active chemicals that change the user's mood. The purpose of this article is to develop and analyze a mathematical model that can be used to understand the dynamics of chewing Khat. The proposed model monitors the dynamics of five compartments, namely, a group of people who do not chew Khat, designated as $N(t)$; a group of people who are surrounded by Khat chewers but do not chew at present and may chew Khat in the future, denoted this as $\Sigma(t)$; a group of people who chew Khat, which is represented in $C(t)$; a group of people contains individuals who consumed Khat quite temporarily for social, spiritual, and recreational purposes, and we describe this group in $T(t)$; and a group of people those who constantly chew Khat, and they are denoted by $H(t)$. We determined the Khat chewing generation number $R_{c 0}$ using the next-generation matrix method, and we have examined the biological meaningfulness, mathematical wellposedness, and stability of both Khat chewing-free and Khat chewing-present equilibrium points of the model analytically. Numerical simulations were presented by solving our dynamical system using Matlabode45 to check the analytical results by considering parameter estimations. The results of this study show that, for $R_{c 0}=.00039$, the Khat chewing-free equilibrium point is stable, and it is unstable for $R_{c 0}=1.194$, and the Khat chewing-present equilibrium point is stable if $R_{c 0}=1.194$, and it is unstable if $R_{c 0}=.00039$. The stability of both equilibrium points implies that, for a high rate of conversion from non-Khat chewer to exposed groups $(\rho)$, the inflow of an insignificant number of Khat chewers to the community produces a significant number of Khat chewers, and if the return back from Khat chewing to the exposed group because of socio-economic, environmental, and religious influences $\left(\alpha_{2}\right)$ grows exponentially, the inflow of an insignificant number of Khat chewers to the community produces an insignificant number of Khat chewers. It is found that increasing the rate of conversion from non-Khat chewer to exposed groups $(\rho)$ makes the disease eradication more challenging. We, therefore, strongly urge religious leaders, social committee leaders, elders, and health experts to teach their followers to reduce their Khat-chewing habits.
\end{abstract}

\section{Introduction}

Khat has different names in different countries. For example, chat in Ethiopia, Qat in Yemen [1], Miraa in Kenya, and Jaad in Somalia [2]. Khat is also known as the "Leaf of Allah," [3] and others express it as a" flower of paradise" [4]. Khat (Catha Edulis) is a wild green leaf and greenish plant that grows commercially in East Africa and the Arabian Peninsula whose branches and leaves are chewed to release liquids having the active chemicals cathinone and cathine, which alter the user's mood $[4,5]$. Chewing Khat has a long history dating back to the early $14^{\text {th }}$ century and is commonly used for social and spiritual purposes [6]. Although initially Muslim prayers used Khat for ceremonies, it is also widely used by Christian followers for recreational purposes [7]. Ethiopia is currently a growing producer of Khat [8-10]. Land for Khat production from 2001/2002 to 2014/2015 increased by $160 \%$, and new areas of the country, which had not been used before, cultivated rapidly [11]. It plays a significant role in the social life of many people in most parts of Ethiopia and is economically beneficial to producers.

The practice of chewing Khat in the Horn of Africa and the Arabian Peninsula has been a popular practice for centuries. Particularly in Ethiopia, the number of Khat users has increased dramatically in recent years, with an average distribution of $15.3 \%[12,13]$. In the new era, for example, in Harar, even children under the age of five harvest Khat [2]. However, the average age of Khat users in Dessie, Ethiopia, is 
19.23 years [14]. Regular users of this plant will find comfort, care, and a feeling of joy. Postchewing effects are often accompanied by sleeplessness, irritability, and poor concentration [4]. Excessive consumption of Khat can lead to serious family, health, and financial problems [15, 16]. Unfortunately, this practice has spread in the Western countries $[17,18]$ and has become a global problem [19].

Chewing Khat is now a public health problem, including heart failure and high blood pressure. At present, Khat chewing is a means for disseminating COVID 19 for the reason that, during harvesting, transporting, distributing, selling, and selecting a package, of course, the communal and individual act of Khat is exposed to human contact, and most of the consumers are chewing unwashed (contaminated) leaves of Khat in public places [20].

The prevalence of chewing Khat is high among high school and college students due to peer pressure, family chewing habits, and living conditions. Many researchers say that a complete ban on the production and trade of Khat could harm Ethiopia's economy in the short term [21]; some say the Ethiopian government has imposed two taxes at the local and federal levels. According to the review, chewing habits have affected the majority of productive age groups, confirmed by many students [7].

According to Dhiraj Kumar Das et al. [22], media awareness is a very effective tool to generate preventive control measures of communicable diseases. To account for the effect of media awareness, there are two approaches: updating the disease transmission rate to accumulate the significant fall in transmission due to preventive measures and by incorporating a mass media compartment to represent the public interaction with mass media.

Mathematical modeling plays an important role in understanding the complexities of communicable diseases such as TB, ebola, rabies, influenza, West Nile virus, zika, measles, pertussis, and HIV/AIDS. The primary purpose of studying communicable disease modeling is to design better health measures to control and ultimately to exterminate the disease. In the last few decades, both mathematicians and epidemiologists have developed many mathematical models to analyze the transmission procedure of several infectious diseases. To determine appropriate public health measures, a threshold quantity, called the basic reproduction number $R_{0}$, plays a significant role. In general, if $R_{0}<1$, the disease will die out in time whereas if $R_{0}>1$, the disease will persist and thus transmit through the individuals. Such type of characterization is familiar in most of the epidemiological models. [23-25].

As stated by Samui et al. [26], mathematical modeling can aid in understanding the following: how transmissible the disease is, when the infectivity becomes high during the course of an epidemic, how acute the disease is, and how effective interventions has been and ought to be.

Makembo, Karandzha, and David [5] developed a mathematical model of Khat (Miraa) chewing, by dividing the entire population into three parts: the number of susceptible persons $S(t)$, the number of light users $L(t)$, and the number of addicts $A(t)$, and they used the SIS compartment model.
We proposed here a deterministic ordinary differential equation model that can represent the dynamics of Khatchewing communicable diseases, and its control mechanisms in public health and socio-economic crises of a country.

It may be useful to assess the effectiveness of control strategies and to study the Khat-chewing methods used to predict Khat-chewing patterns. We stratified the total human population into five compartments, namely, a group of people who do not chew Khat, designated by $N(t)$, a group of people who are surrounded by Khat chewers but do not chew at present; they may chew Khat in the future, and we denote this as $\Sigma(t)$, a group of people who chew Khat, which we represent in $C(t)$, a group of people containing individuals who consumed Khat quite temporarily for social, spiritual, and recreational purposes, and we describe this group in $T(t)$, and a group of people those who constantly chew Khat, and they are denoted by $H(t)$, and the general population is denoted by $Z(t)$ and is expressed as $Z(t)=N(t)+\Sigma(t)+C(t)+T(t)+\mathrm{H}(t)$. We also note that people who chew Khat may return to vulnerable groups due to social, economic, environmental, and religious factors with a rate $\alpha_{2}$ and constant and diverse population. In this study, we used a compartment model similar to SEIR [22-29]. In this deterministic model, we have considered a system of first-order nonlinear ordinary differential equations, which is derived and analyzed analytically and analyzed only numerically using Matlab ode45. From our dynamical system, we got the free equilibrium point of Khat chewing, that is, the point where Khat chewing is not practiced in a given population, and Khat chewing presents equilibrium points and the Khatchewing generation number, denoted by $R_{c 0}$. The generation number $R_{c 0}$ is intended to determine whether the chewing habits of the population have disappeared or increased.

The simulations of our model were performed using parameters with their estimated values. Through simulations, the model is analyzed to obtained different situations that produce interesting results among specific classes. The article is organized as follows; in the second section, we formulate a Khat-chewing model, by classifying a constant and heterogeneous entire population into five groups; in the third section, we analyzed our model, checked its biological completeness and mathematically correct significance, determined equilibrium points and the Khat-chewing generation number, and tested existence and stability of equilibrium points of the dynamical system. In section four, we have presented numerical simulations that support our results. Finally, we conclude the manuscript with a conclusion and recommendation in the fifth section.

\section{Model Formulation for Khat Chewing}

Our model contains five first-order nonlinear ordinary differential equations. The parameters we used in our study are all positive and ranges between 0 and 1 and are listed and described in Table 1, and our models' diagram representation is shown in Figure 1. 
TABle 1: Parameters and their descriptions.

\begin{tabular}{lcc}
\hline No. & Parameters & Description \\
\hline 1 & $\epsilon$ & Natural birth rate \\
2 & $\varepsilon$ & Natural death rate \\
3 & $\rho$ & Rate of conversion from non-Khat chewer to the exposed group \\
4 & $\alpha 1$ & Rate of conversion from the exposed group to Khat chewer \\
5 & $\alpha 2$ & Rate of return back from Khat chewer to exposed because of socio-economic, environmental, and religious factors \\
6 & $\alpha 3$ & Rate of removal from Khat chewing to temporary Khat chewers and regular Khat chewers. \\
7 & $\alpha 4$ & Rate of return back from temporarily Khat chewers to the Khat chewers group \\
8 & $\omega$ & The probability of conversion from Khat chewing to temporarily Khat chewers \\
9 & $1-\omega$ & The probability of conversion from Khat chewing to regular Khat chewers. \\
10 & $\delta$ & Rate of conversion from temporarily chewers to permanent chewers \\
11 & $v$ & Khat-chewing induced disease death rate
\end{tabular}

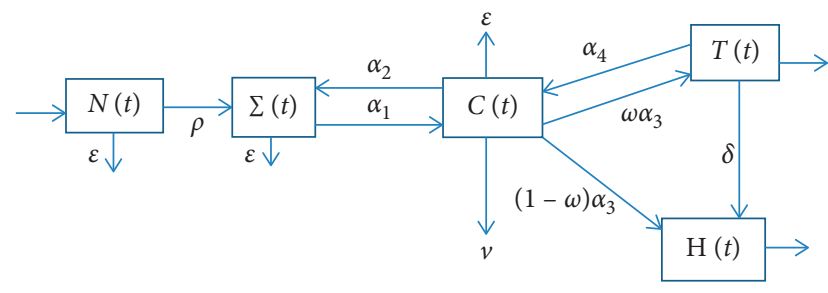

FIgURE 1: Flow chart of the Khat-chewing model.

We symbolized the entire population using a flow chart as follows.

The mathematical representation of the above flow chart is described as follows:

$$
\begin{aligned}
\frac{\mathrm{d} N(t)}{\mathrm{d} t}=\varepsilon Z(t)-\varepsilon N(t)-\frac{\rho \sum(t) N(t)}{Z(t)} & \\
\frac{\mathrm{d} \Sigma(t)}{\mathrm{d} t}= & \frac{\rho \sum(t) N(t)}{Z(t)}-\varepsilon \Sigma(t)-\alpha_{1} \Sigma(t)+\alpha_{2} C(t), \\
\frac{\mathrm{d} C(t)}{\mathrm{d} t}= & \alpha_{1} \sum(t)+\alpha_{4} T(t)-\varepsilon C(t) \\
& -\alpha_{3} C(t)-\alpha_{2} C(t)-v C(t), \\
\frac{\mathrm{d} T(t)}{\mathrm{d} t}= & \alpha_{3} \omega C(t)-\alpha_{4} T(t)-\delta T(t)-\varepsilon T(t), \\
\frac{\mathrm{d} H(t)}{\mathrm{d} t}= & \alpha_{3}(1-\omega) C(t)+\delta T(t)-\varepsilon \mathrm{H}(t) .
\end{aligned}
$$

Subject to the initial conditions, $N(0)=N_{0}, \Sigma(0)=\Sigma_{0}$, $C(0)=C_{0}, T(0)=T_{0}$, and $H(0)=H_{0}$.

\section{Model Analysis}

\subsection{Positivity of Model Solutions}

Theorem 1. If initial conditions of (1) are all positive, then it has a positive solution.

Proof. Suppose $N(0)=N_{0}>0, \Sigma(0)=\Sigma_{0}>0, C(0)=C_{0}$ $>0, T(0)=T_{0}>0$, and $H(0)=H_{0}>0$, then

(i) $\mathrm{d} N(t) / \mathrm{d} t=\epsilon Z(t)-\varepsilon N(t)-(\rho \Sigma(t) N(t) / Z(t)) \geq-$ $\varepsilon N(t)-(\rho \Sigma(t) N(t) / Z(t))$

$$
\begin{aligned}
& \mathrm{d} N(t) / \mathrm{d} t \geq-N(t)\left(\varepsilon+\rho \sum(t) / Z(t)\right) \\
& \mathrm{d} N(t) / N(t) \geq-\left(\varepsilon+\rho \sum(t) / Z(t)\right) \mathrm{d} t \\
& \ln (N(t)) \geq-\int_{0}^{t} \varepsilon+\rho \sum(t) / Z(t) \mathrm{d} t+c_{1} \\
& N(t) \geq N_{0} e^{-} \int_{0}^{\varepsilon+\rho \Sigma(t) / Z(t) \mathrm{d} t}>0
\end{aligned}
$$

Hence, the solution of $N(t)$ is positive.

(ii) $\mathrm{d} \Sigma(t) / \mathrm{d} t=\left(\rho \sum(t) N(t) / Z(t)\right)-\varepsilon \Sigma(t)-\alpha_{1} \Sigma(t)+$ $\alpha_{2} C(t) \geq-\left(\varepsilon \Sigma(t)+\alpha_{1} \Sigma(t)\right)$

$$
\begin{aligned}
& \mathrm{d} \Sigma(t) / \Sigma(t) \geq-\left(\varepsilon+\alpha_{1}\right) \mathrm{d} t \Rightarrow \ln (\Sigma(t)) \geq-\int_{0}^{t} \\
& \left(\varepsilon+\alpha_{1}\right) \mathrm{d} t+c_{2} \Rightarrow \Sigma(t) \geq e^{-\int_{0}^{t}\left(\varepsilon+\alpha_{1}\right) \mathrm{d} t+c_{2}} \\
& \Sigma(t) \geq \Sigma_{0} e^{-\left(\varepsilon+\alpha_{1}\right) t}>0
\end{aligned}
$$

Hence, the solution of $\Sigma(t)$ is positive.

(iii) $\mathrm{d} C(t) / \mathrm{d} t=\alpha_{1} \Sigma(t)+\alpha_{4} T(t)-\varepsilon C(t)-\alpha_{3} \omega C(t)-$ $\alpha_{3}(1-\omega) C(t)-\alpha_{2} C(t)-v C(t)$

$$
\begin{aligned}
& \mathrm{d} C(t) / \mathrm{d} t \geq C(t)\left(-\varepsilon-\alpha_{3}-\alpha_{2}-v\right) \\
& \mathrm{d} C(t) / C(t) \geq\left(-\varepsilon-\alpha_{3}-\alpha_{2}-v\right) \mathrm{d} t \\
& \ln (C(t)) \geq-\int_{0}^{t}\left(\varepsilon+\alpha_{\beta^{t}}+\alpha_{2}\right. \\
& \left.+v) \mathrm{~d} t+c_{3} \Rightarrow C(t) \geq e^{-} \int_{0}+\alpha_{3}+v\right) \mathrm{d} t+c_{3} \\
& C(t) \geq C_{0} e^{-\left(\varepsilon+\alpha_{3}+\alpha_{2}+v\right) t}>0
\end{aligned}
$$

Hence, the solution of $C(t)$ is positive.

Using a similar method, solutions of $T(t)$ and $H(t)$ are positive.

Since all solutions of the dynamical system are positive, the feasible solution containing all solutions of (1) has the form:

$$
\mathscr{H}=\left\{N(t), \Sigma(t), C(t), T(t), H(t) \in \mathbb{R}^{+5}\right\}
$$

3.2. Boundedness of Solutions. To say that the dynamical system (1) is biologically complete and mathematically correct, all its solutions must be bounded.

Theorem 2. For all $t>0$, a region $\mathscr{H}$ exists in which all solutions of (1) are contained and bounded.

Proof. The rate of change of total population size $Z(t)$ is defined as follow:

$$
\frac{\mathrm{d} Z(t)}{\mathrm{d} t}=\frac{\mathrm{d} N(t)}{\mathrm{d} t}+\frac{\mathrm{d} \Sigma(t)}{\mathrm{d} t}+\frac{\mathrm{d} C(t)}{\mathrm{d} t}+\frac{\mathrm{d} T(t)}{\mathrm{d} t}+\frac{\mathrm{d} H(t)}{\mathrm{d} t} .
$$


By substituting all values of left-hand sides of (1) in the above rate of change of total solution, we obtained $(\mathrm{d} Z(t) / \mathrm{d} t)=Z(t)(\epsilon-\varepsilon)-v H(t)$; assuming that diseaseinduced death rate $v=0$, initial population size $Z(0)=Z_{0}$, and $\epsilon<\varepsilon$,

$$
\begin{aligned}
& \frac{\mathrm{d} Z(t)}{\mathrm{d} t} \leq Z(t)(\epsilon-\varepsilon)=-Z(t) \tau, \\
& \frac{\mathrm{d} Z(t)}{\mathrm{d} t} \leq-Z(t) \tau .
\end{aligned}
$$

$0 \leq Z(t) \leq Z_{0} e^{-\tau t}$ for all $t>0$; hence, all solutions of (1) are bounded.

\subsection{Existence and Stability of Equilibrium Points}

3.3.1. Scaling. To reduce the complexity of our model, we resize the fraction by scaling it as follows. Assuming that $e(t)=\Sigma(t) / Z(t), \quad c(t)=C(t) / Z(t), \quad \chi(t)=T(t) / Z(t)$, $n(t)=N(t) / Z(t)$, and $h(t)=H(t) / Z(t)$, our dynamical system (1) is reduced to the following:

$$
\begin{aligned}
& \frac{\mathrm{d} n(t)}{\mathrm{d} t}=\epsilon-\varepsilon n(t)-\rho e(t) n(t), \\
& \frac{\mathrm{d} e(t)}{\mathrm{d} t}=\rho e(t) n(t)-\varepsilon e(t)-\alpha_{1} e(t)+\alpha_{2} c(t), \\
& \frac{\mathrm{d} c(t)}{\mathrm{d} t}=\alpha_{1} e(t)+\alpha_{4} \chi(t)-\varepsilon c(t)-\alpha_{3} c(t)-v c(t)-\alpha_{2} c(t), \\
& \frac{\mathrm{d} \chi(t)}{\mathrm{d} t}=\alpha_{3} \omega c(t)-\alpha_{4} \chi(t)-\delta \chi(t)-\varepsilon \chi(t), \\
& \frac{\mathrm{d} h(t)}{\mathrm{d} t}=\alpha_{3}(1-\omega) c(t)+\delta \chi(t)-\varepsilon h(t),
\end{aligned}
$$

subject to the initial conditions, $n(0)=n_{0}, e(0)=e_{0}$, $c(0)=c_{0}, \chi(0)=\chi_{0}$, and $H(0)=H_{0}$.

Since the compartment $h(t)$ does not appear in the first four equations of the model (5), the dynamics of (5) is the same as the following one:

$$
\begin{aligned}
& \frac{\mathrm{d} n(t)}{\mathrm{d} t}=\epsilon-\varepsilon n(t)-\rho e(t) n(t), \\
& \frac{\mathrm{d} e(t)}{\mathrm{d} t}=\rho e(t) n(t)-\varepsilon e(t)-\alpha_{1} e(t)+\alpha_{2} c(t), \\
& \frac{\mathrm{d} c(t)}{\mathrm{d} t}=\alpha_{1} e(t)+\alpha_{4} \chi(t)-\varepsilon c(t)-\alpha_{3} c(t)-v c(t)-\alpha_{2} c(t), \\
& \frac{\mathrm{d} \chi(t)}{\mathrm{d} t}=\alpha_{3} \omega c(t)-\alpha_{4} \chi(t)-\delta \chi(t)-\varepsilon \chi(t) .
\end{aligned}
$$

3.3.2. Equilibrium Points. Khat chewing-free equilibrium points: Khat chewing-free equilibrium point $E_{c *}$ of (1) is a balance point that exists when Khat chewing is not practiced in a given population, that is, if there are no people chewing Khat. We determine Khatchewing-free equilibrium point $E_{c *}^{*} \quad$ of (6) by assuming $e(t)=\chi(t)=c(t)=0$ and substituting it into the right side of (6), and finally, equate the result to zero.

Hence, $\quad E_{c *}^{*}=(n, e, c, \chi)=(1,0,0,0) \quad$ and $E_{c *}=(n, e, c, \chi, h)=(1,0,0,0,0)$.

Khat chewing-present equilibrium points: Khat chewing-present equilibrium point $E_{c *}^{p}$ of (1) is the equilibrium point that exists when Khat chewing is practiced in a given population, that is, if there are people who chew Khat. We determine Khat chewing-present equilibrium point $E_{c *}^{p *}$ of (6) by assuming at least one of the variables, $e(t), \chi(t)$, or $c(t) \neq 0$.

Hence,

$$
\begin{aligned}
E_{c *}^{p *}\left(n_{1}, e_{1}, c_{1}, \chi_{1}\right)= & \left(\begin{array}{c}
1-\frac{c_{1}\left(\alpha_{4}+\delta+\epsilon\right)\left(\epsilon+\alpha_{3}+v+\alpha_{2}\right)\left(\epsilon+\alpha_{1}-\alpha_{2}\right)+\left(\epsilon+\alpha_{1}-\alpha_{2}\right) \alpha_{4} \alpha_{3} \omega c_{1}}{\epsilon\left(\alpha_{4}+\delta+\varepsilon\right)}, \\
\left(\epsilon+\alpha_{3}+v+\alpha_{2}-\frac{\alpha_{3} \alpha_{4} \omega}{\alpha_{4}+\delta+\epsilon}\right) c_{1}, c_{1}, \frac{\alpha_{3} \omega c_{1}}{\alpha_{4}+\delta+\epsilon}
\end{array}\right), \\
E_{c *}^{p}\left(n_{1}, e_{1}, c_{1}, \chi_{1}, h_{1}\right)= & \left(\begin{array}{c}
1-\frac{c_{1}\left(\alpha_{4}+\delta+\epsilon\right)\left(\epsilon+\alpha_{3}+v+\alpha_{2}\right)\left(\epsilon+\alpha_{1}-\alpha_{2}\right)+\left(\epsilon+\alpha_{1}-\alpha_{2}\right) \alpha_{4} \alpha_{3} \omega c_{1}}{\epsilon\left(\alpha_{4}+\delta+\epsilon\right)}, \\
\left(\epsilon+\alpha_{3}+v+\alpha_{2}-\frac{\alpha_{3} \alpha_{4} \omega}{\alpha_{4}+\delta+\epsilon}\right) c_{1}, c_{1}, \frac{\alpha_{3} \omega c_{1}}{\alpha_{4}+\delta+\epsilon}, \frac{c_{1} \alpha_{3}(1-\omega)\left(\alpha_{4}+\delta+\epsilon\right)+\alpha_{3} \omega c_{1}}{\epsilon\left(\alpha_{4}+\delta+\epsilon\right)}
\end{array}\right) .
\end{aligned}
$$

3.3.3. Khat-Chewing Generation Number $\left(\mathbf{R}_{\mathrm{c} 0}\right)$. The Khatchewing generation number $\left(R_{c 0}\right)$ is used to determine secondary Khat chewers, i.e., one Khat chewer individual would create another chewer over the duration of the
Khat-chewing period, as long as everyone else is exposed to Khat chewers. The Khat-chewing generation number plays an important role in controlling the significant increase in the number of Khat chewers. In a more general 
way, the Khat-chewing generation number $R_{c 0}$ can be stated as the number of new infections (new Khat chewers) created by a typical infective population at a disease-free equilibrium. $R_{c 0}<1$ determines, on average, an infected population creates less than one new infected population during the course of its infective period, and the infection can die out but for $R_{c 0}=1$, an infected individual can infect on an average 1 person, that is, the spread of the disease is stable. In the reverse way, $R_{c 0}>1$ determines each infected population creates, on average, more than one new infection, and the disease can spread over the population $[26,29,30]$. To determine the Khatchewing generation number, we used the production matrix method described in [26, 29-33].

Afterwards, we determine the square matrix $\mathscr{M}$, which is the emergence rate of new Khat-chewing devices (new infections), and $\delta$ which is the transmission rate of individuals. The order of both matrices is $n x n$, where, $n$ is the number of infected compartments. The new infected compartment and transfer compartments are $e(t)$ and $c(t)$, respectively, and we get a new subsystem.

$$
\begin{aligned}
\frac{\mathrm{d} e(t)}{\mathrm{d} t} & =\rho e(t) n(t)-\epsilon e(t)-\alpha_{1} e(t)+\alpha_{2} c(t), \\
\frac{\mathrm{d} c(t)}{\mathrm{d} t} & =\alpha_{1} e(t)+\alpha_{4} \chi(t)-\epsilon c(t)-\alpha_{3} c(t)-v c(t)-\alpha_{2} c(t), \\
\left(\begin{array}{c}
\frac{\mathrm{d} e(t)}{\mathrm{d} t} \\
\frac{\mathrm{d} c(t)}{\mathrm{d} t}
\end{array}\right) & =\left(\begin{array}{c}
\rho e(t) n(t) \\
0
\end{array}\right)-\left(\begin{array}{c}
\epsilon e(t)+\alpha_{1} e(t)-\alpha_{2} c(t) \\
-\alpha_{1} e(t)-\alpha_{4} \chi(t)+\varepsilon c(t)+\alpha_{3} c(t)+v c(t)+\alpha_{2} c(t)
\end{array}\right) .
\end{aligned}
$$

$\widetilde{O}=\left(\begin{array}{cc}\epsilon+\alpha_{1} & -\alpha_{2} \\ -\alpha_{1} & \epsilon+\alpha_{3}+\alpha_{2}+v\end{array}\right)\left(\begin{array}{cc}\rho n(t) & 0 \\ 0 & 0\end{array}\right)$

and
Because $\widetilde{\sigma}$ is nonsingular, $\mho^{-1}$ is also nonsingular. $\mathscr{M} \mho^{-1}$ is called the next-generation matrix of our model, and its maximum positive Eigen value is known as the Khatchewing generation number:

$$
\begin{gathered}
\sigma^{-1}=\frac{1}{\left(\left(\epsilon+\alpha_{1}\right)\left(\epsilon+\alpha_{3}+\alpha_{2}+v\right)-\alpha_{2} \alpha_{1}\right)}\left(\begin{array}{cc}
\epsilon+\alpha_{3}+\alpha_{2}+v & \alpha_{2} \\
\alpha_{1} & \epsilon+\alpha_{1}
\end{array}\right), \\
M \sigma^{-1}=\frac{1}{\left(\left(\epsilon+\alpha_{1}\right)\left(\epsilon+\alpha_{3}+\alpha_{2}+v\right)-\alpha_{2} \alpha_{1}\right)}\left(\begin{array}{cc}
\left(\epsilon+\alpha_{3}+\alpha_{2}+v\right) \rho n(t) & \alpha_{2} \rho n(t) \\
0 & 0
\end{array}\right) .
\end{gathered}
$$

The eigenvalues are 0
$\left.+\alpha_{3}+\alpha_{2}+v\right) \rho n(t) /\left(\left(\epsilon+\alpha_{1}\right)\left(\epsilon+\alpha_{3}+\alpha_{2}+v\right)-\alpha_{2} \alpha_{1}\right)$.

The maximum positive eigenvalue of $\mathscr{M} \mho^{-1}$ which is the Khat-chewing generation number is

$$
R_{c 0}=\frac{\left(\epsilon+\alpha_{3}+\alpha_{2}+v\right) \rho n(t)}{\left(\epsilon+\alpha_{1}\right)\left(\epsilon+\alpha_{3}+\alpha_{2}+v\right)-\alpha_{2} \alpha_{1}} .
$$

If $\left(\epsilon+\alpha_{1}\right)\left(\epsilon+\alpha_{3}+\alpha_{2}+v\right)-\alpha_{2} \alpha_{1}>\left(\epsilon+\alpha_{3}+\alpha_{2}+v\right)$ $\rho n(t)$, then $R_{c 0}<1, R_{c 0}<1$ implies no Khat chewers or the number of Khat chewers reduced to zero [26, 29, 30].
If $\quad\left(\epsilon+\alpha_{1}\right)\left(\epsilon+\alpha_{3}+\alpha_{2}+v\right)-\alpha_{2} \alpha_{1}<\left(\epsilon+\alpha_{3}+\alpha_{2}+v\right)$ $\rho n(t)$, then $R_{c 0}>1, R_{c 0}>1$ which indicates that the insignificant inflow of Khat chewers into the community will produce a significant number of Khat chewers [26, 29, 30].

Stability analysis of the Khat chewing-free equilibrium point (analytically): the Jacobian matrix of (6) is defined as follows:

$$
J(n, e, c, \chi)=\left(\begin{array}{cccc}
-\epsilon-\rho e(t) & -\rho n(t) & 0 & 0 \\
\rho e(t) & \rho n(t)-\epsilon-\alpha_{1} & \alpha_{2} & 0 \\
0 & \alpha_{1} & -\epsilon-\alpha_{3}-\nu-\alpha_{2} & \alpha_{4} \\
0 & 0 & \alpha_{3} \omega & -\alpha_{4}-\delta-\epsilon
\end{array}\right)
$$


The Jacobian matrix of (6) at Khat chewing-free equilibrium point $E_{c *}^{*}=(1,0,0,0)$ is as follows:

$$
J(1,0,0,0)=\left(\begin{array}{cccc}
-\epsilon & -\rho & 0 & 0 \\
0 & \rho-\epsilon-\alpha_{1} & \alpha_{2} & 0 \\
0 & \alpha_{1} & -\epsilon-\alpha_{3}-\nu-\alpha_{2} & \alpha_{4} \\
0 & 0 & \alpha_{3} \omega & -\alpha_{4}-\delta-\epsilon
\end{array}\right) .
$$

Theorem 3. The Khat chewing-free equilibrium point $E_{c *}$ of (1) is stable if $R_{c 0}<1$, and it is unstable if $R_{c 0}>1$.
Proof. Proving local stability of $E_{c *}^{*}$ leads us to proving local stability of $E_{c *}$.

The characteristics equation $J(1,0,0,0)$ is determined using $\left|I_{4}\right|=0$ :

$$
\left|\begin{array}{cccc}
-\epsilon-\theta & -\rho & 0 & 0 \\
0 & \rho-\epsilon-\alpha_{1}-\theta & \alpha_{2} & 0 \\
0 & \alpha_{1} & -\epsilon-\alpha_{3}-\nu-\alpha_{2}-\theta & \alpha_{4} \\
0 & 0 & \alpha_{3} \omega & -\alpha_{4}-\delta-\epsilon-\theta
\end{array}\right|=0 ;
$$

$(-\theta)\left(-\theta^{3}+\aleph_{1} \theta^{2}+\aleph_{2} \theta+\aleph_{3}\right)=0$, where

$$
\begin{aligned}
& \aleph_{1}=\left[\left(\rho-\alpha_{1}-\epsilon\right)+\left(-\alpha_{3}-\nu-\alpha_{2}-\epsilon\right)+\left(-\alpha_{4}-\delta-\epsilon\right)\right], \\
& \aleph_{2}=\left[-\left(\rho-\alpha_{1}-\epsilon\right)\left(-\alpha_{3}-\nu-\alpha_{2}-\epsilon\right)-\left(\rho-\alpha_{1}-\epsilon\right)\left(-\alpha_{4}-\delta-\epsilon\right)-\left(-\alpha_{3}-\nu-\alpha_{2}-\epsilon\right)\left(-\alpha_{4}-\delta-\epsilon\right)+\alpha_{1} \alpha_{2}\right], \\
& \aleph_{3}=-\left(\rho-\alpha_{1}-\epsilon\right)-\alpha_{4} \alpha_{3} \omega+\left(\rho-\alpha_{1}-\epsilon\right)\left(-\alpha_{3}-\nu-\alpha_{2}-\epsilon\right)\left(-\alpha_{4}-\delta-\epsilon\right)-\left(-\alpha_{4}-\delta-\epsilon\right) \alpha_{1} \alpha_{2}+\alpha_{4} \alpha_{3} \omega .
\end{aligned}
$$

The eigenvalues are $\theta_{1}=-\varepsilon$, and we can find roots of $-\theta^{3}+\aleph_{1} \theta^{2}+\aleph_{2} \theta+\aleph_{3}=0$ using the Routh-Herwitz stability criterion.

Definition 1. The Routh-Herwitz stability criterion [34] states the following:

(a) If there is no sign change in the first column of the Routh-Herwitz table, the system is stable.

(b) The number of roots of a polynomial lying in the right half-plane is equal to the number of sign changes in the first column. Consequently, the system is unstable if the poles are on the right.
If $\aleph_{1}<0,\left(\aleph_{1} \aleph_{2}+\aleph_{3} / \aleph_{3}\right)<0$ and $\aleph_{3}<0$, then there are no sign changes in the first column of the Routh-Herwitz table. Using the above definition, the system is stable; this implies that $R_{c 0}<1$ (the Jacobian matrix has a negative eigenvalue if $R_{c 0}<1$ ), and $E_{c *}^{*}$ is stable. Hence, $E_{c *}$ is stable.

If at least one of $\aleph_{1},\left(\aleph_{1} \aleph_{2}+\aleph_{3} / \aleph_{3}\right)$ or $\aleph_{3}$ are positive, there is a sign change in the first column of the Routh-Herwitz table. Then, the system is unstable; this implies that $R_{c 0}>1$, and $E_{c *}^{*}$ is unstable. Hence, $E_{c *}$ is unstable.

Stability analysis of the Khat chewing-present equilibrium point (analytically): the Jacobian matrix of (6) is defined as follows:

$$
J(n, e, c, \chi)=\left(\begin{array}{cccc}
-\epsilon-\rho e(t) & -\rho n(t) & 0 & 0 \\
\rho e(t) & \rho n(t)-\epsilon-\alpha_{1} & \alpha_{2} & 0 \\
0 & \alpha_{1} & -\epsilon-\alpha_{3}-\nu-\alpha_{2} & \alpha_{4} \\
0 & 0 & \alpha_{3} \omega & -\alpha_{4}-\delta-\epsilon
\end{array}\right) .
$$

The Jacobian matrix of (6) at Khat chewing-present equilibrium point $E_{c *}^{p *}$ is

$$
J\left(n_{1}, e_{1}, c_{1}, \chi_{1}\right)=\left(\begin{array}{cccc}
-\epsilon-\rho e_{1} & -\rho n_{1} & 0 & 0 \\
\rho e_{1} & \rho n_{1}-\epsilon-\alpha_{1} & \alpha_{2} & 0 \\
0 & \alpha_{1} & -\epsilon-\alpha_{3}-\nu-\alpha_{2} & \alpha_{4} \\
0 & 0 & \alpha_{3} \omega & -\alpha_{4}-\delta-\epsilon
\end{array}\right) \text {, }
$$


where

$$
\begin{aligned}
& n_{1}=1-\frac{c_{1}\left(\alpha_{4}+\delta+\epsilon\right)\left(\epsilon+\alpha_{3}+v+\alpha_{2}\right)\left(\epsilon+\alpha_{1}-\alpha_{2}\right)+\left(\epsilon+\alpha_{1}-\alpha_{2}\right) \alpha_{4} \alpha_{3} \omega c_{1}}{\epsilon\left(\alpha_{4}+\delta+\epsilon\right)}, \\
& e_{1}=\left(\epsilon+\alpha_{3}+v+\alpha_{2}-\frac{\alpha_{3} \alpha_{4} \omega}{\alpha_{4}+\delta+\epsilon}\right) c_{1} .
\end{aligned}
$$

Theorem 4. The Khat chewing-present equilibrium point $E_{c *}^{p}$ of (1) is stable if $R_{c 0}>1$ and unstable if $R_{c 0}<1$.
Proof. Proving stability of $E_{c *}^{p *}$ leads us to proving local stability of $E_{c *}^{p}$.

The characteristics equation of the Jacobian matrix of (6) at $E_{c *}^{p *}$ is

$$
\left|\begin{array}{cccc}
-\epsilon-\rho e_{1}-\theta & -\rho n_{1} & 0 & 0 \\
\rho e_{1} & \rho n_{1}-\epsilon-\alpha_{1}-\theta & \alpha_{2} & 0 \\
0 & \alpha_{1} & -\epsilon-\alpha_{3}-\nu-\alpha_{2}-\theta & \alpha_{4} \\
0 & 0 & \alpha_{3} \omega & -\alpha_{4}-\delta-\epsilon-\theta
\end{array}\right|=0
$$

$\theta^{4}+\aleph_{4} \theta^{3}+\aleph_{5} \theta^{2}+\aleph_{6} \theta+\aleph_{7}=0$, where

$$
\begin{aligned}
\aleph_{4}= & -\rho n_{1}+\alpha_{1}-\alpha_{3}-v-\alpha_{2}+\alpha_{4}-\epsilon-\rho e_{1}, \\
\aleph_{5}= & \left(\rho e_{1}\right)^{2}+\left(\rho n_{1}-\epsilon-\alpha_{1}\right)\left(-\epsilon-\rho e_{1}\right)+\left(-\epsilon-\rho e_{1}\right)\left(-\epsilon-\alpha_{3}-v-\alpha_{2}\right)+\left(\rho n_{1}-\epsilon-\alpha_{1}\right)\left(-\epsilon-\alpha_{3}-v-\alpha_{2}\right)+\left(-\epsilon-\rho e_{1}\right)\left(-\epsilon-\alpha_{4}-\delta\right) \\
& +\left(\rho n_{1}-\epsilon-\alpha_{1}\right)\left(-\epsilon-\alpha_{4}-\delta\right)+\left(-\epsilon-\alpha_{3}-v-\alpha_{2}\right)\left(-\epsilon-\alpha_{4}-\delta\right)+\left(-\epsilon-\rho e_{1}\right) \alpha_{4}-\alpha_{1} \alpha_{2}+\left(\rho n_{1}-\epsilon-\alpha_{1}\right) \alpha_{4}, \\
\aleph_{6}= & -\left(\rho e_{1}\right)^{2}\left(-\epsilon-\alpha_{3}-v-\alpha_{2}\right)-\alpha_{4}\left(\rho e_{1}\right)^{2}-\left(\rho e_{1}\right)^{2}\left(-\epsilon-\alpha_{4}-\delta\right)+\left(-\epsilon-\alpha_{4}-\delta\right) \alpha_{2} \alpha_{1}-\left(-\epsilon-\rho e_{1}\right)\left(\rho n_{1}-\epsilon-\alpha_{1}\right)\left(-\epsilon-\alpha_{3}-v-\alpha_{2}\right) \\
& -\left(-\epsilon-\rho e_{1}\right)\left(\rho n_{1}-\epsilon-\alpha_{1}\right)\left(-\epsilon-\alpha_{4}-\delta\right)-\left(-\epsilon-\rho e_{1}\right)\left(-\epsilon-\alpha_{4}-\delta\right)\left(-\epsilon-\alpha_{4}-\delta\right)-\left(-\epsilon-\rho e_{1}\right)\left(-\epsilon-\alpha_{4}-\delta\right)\left(-\epsilon-\alpha_{4}-\delta\right) \\
& -\left(\rho n_{1}-\epsilon-\alpha_{1}\right)\left(-\epsilon-\alpha_{4}-\delta\right)\left(-\epsilon-\alpha_{3}-v-\alpha_{2}\right)+\left(-\epsilon-\rho e_{1}\right) \alpha_{1} \alpha_{2}-\left(-\epsilon-\rho e_{1}\right)\left(\rho n_{1}-\epsilon-\alpha_{1}\right) \alpha_{4}, \\
\aleph_{7}= & \left(\rho e_{1}\right)^{2}\left(-\epsilon-\alpha_{3}-v-\alpha_{2}\right)\left(-\epsilon-\alpha_{4}-\delta\right)+\left(-\epsilon-\rho e_{1}\right)\left(-\epsilon-\alpha_{3}-v-\alpha_{2}\right)\left(\rho n_{1}-\epsilon-\alpha_{1}\right)\left(-\epsilon-\alpha_{4}-\delta\right)-\left(-\epsilon-\rho e_{1}\right)\left(-\epsilon-\alpha_{4}-\delta\right) \alpha_{1} \alpha_{2} .
\end{aligned}
$$

If $\aleph_{4}>0,\left(\aleph_{4} \aleph_{5}-\aleph_{6} / \aleph_{4}\right)>0, \aleph_{7}>0$, and $\left(\left(\aleph_{4} \aleph_{5} \aleph_{6}-\right.\right.$ $\left.\left.\aleph_{6}^{2}-\aleph_{7} \aleph_{6}\right) \aleph_{4} / \aleph_{4} \aleph_{5}-\aleph_{6}\right)>0$, or all are negative, then there are no sign changes in the first column of the Routh-Herwitz table; hence, the system is stable; this implies that $R_{c 0}<1$, and $E_{c *}^{p *}$ is unstable. Therefore, $E_{c *}^{p}$ is unstable.

If at least one of $\aleph_{4},\left(\aleph_{4} \aleph_{5}-\aleph_{6} / \aleph_{4}\right), \aleph_{7}$, and $\left(\left(\aleph_{4}\right.\right.$ $\left.\left.\aleph_{5} \aleph_{6}-\aleph_{6}^{2}-\aleph_{7} \aleph_{6}\right) \aleph_{4} / \aleph_{4} \aleph_{5}-\aleph_{6}\right)$ are negative or positive, there are sign changes in the first column of the Routh-Herwitz table. Then, the system is unstable; this implies that $R_{c 0}>1$, and $E_{c *}^{p *}$ is stable. Hence, $E_{c *}^{p}$ is stable.

\section{Model Numerical Simulation}

4.1. Parameter Estimation and Stability Analysis of Equilibrium Points Numerically. Table 2 shows the estimated and recommended parameter values and their corresponding Khat-chewing generation numbers. Using the results, we analyzed our model numerically.
4.2. Stability Analysis of Khat Chewing-Free Equilibrium Point $E_{c *}^{*}=(1,0,0,0)$. We analyzed the stability of Khat chewing-free equilibrium point $E_{c *}^{*}=(1,0,0,0)$ using estimated values of column 1 and 4 of Table 2 as follows.

For column 1,

$$
J(1,0,0,0)=\left(\begin{array}{cccc}
-0.3234 & -0.00015 & 0 & 0 \\
0 & -0.38825 & 0.0075 & 0 \\
0 & 0.065 & -1.1309 & 0.79 \\
0 & 0 & 0.1575 & -1.7034
\end{array}\right) \text {. }
$$

The characteristics equation of $J(1,0,0,0)$ is determined using $\left|I_{4}\right|=0$; then, its characteristic's equation is

$$
\theta^{4}+3.55 \theta^{3}+3.94 \theta^{2}+1.64 \theta+0.23=0 .
$$


TABLE 2: Estimated values of parameters and their corresponding Khat-chewing generation numbers.

\begin{tabular}{|c|c|c|c|c|c|}
\hline Parameters & Value 1 & Value 2 & Value 3 & Value 4 & Source \\
\hline$\epsilon$ & 0.3234 & 0.3234 & 0.3234 & 0.3234 & WB \\
\hline$\varepsilon$ & 0.06551 & 0.06551 & 0.06551 & 0.06551 & {$[5,35]$} \\
\hline$\rho$ & 0.00015 & 0.00038 & 0.055 & 0.9 & Estimated \\
\hline$\alpha 1$ & 0.065 & 0.07 & 0.65 & 0.68 & Estimated \\
\hline$\alpha 2$ & 0.0075 & 0.0085 & 0.25 & 0.65 & Estimated \\
\hline$\alpha 3$ & 0.35 & 0.35 & 0.35 & 0.35 & Estimated \\
\hline$\alpha 4$ & 0.79 & 0.79 & 0.79 & 0.79 & Estimated \\
\hline$\omega$ & 0.45 & 0.45 & 0.45 & 0.45 & Estimated \\
\hline $1-\omega$ & 0.65 & 0.65 & 0.65 & 0.65 & Estimated \\
\hline$\delta$ & 0.59 & 0.59 & 0.59 & 0.59 & Estimated \\
\hline$v$ & 0.45 & 0.45 & 0.45 & 0.45 & Estimated \\
\hline$n$ & 1000 & 1000 & 1000 & 1000 & Estimated \\
\hline & $R_{\mathrm{c} 0}=0.39$ & $R_{\mathrm{c} 0}=0.97$ & $R_{\mathrm{c} 0}=64.32$ & $R_{\mathrm{c} 0}=1193.76$ & \\
\hline At $E^{\mathcal{C} *}$ & $R_{\mathrm{c} 0}(1,0,0,0,0)=0.00039$ & $R_{\mathrm{c} 0}(1,0,0,0,0)=0.00097$ & $R_{\mathrm{c} 0}(1,0,0,0,0)=0.064$ & $R_{\mathrm{c} 0}(1,0,0,0,0)=1.194$ & \\
\hline
\end{tabular}

$\mathrm{WB}=$ World Bank (2017, 2018, and 2019).

The eigenvalues are $-1.8912-0.9460-0.3564+$ $0.0392 i$ and $-0.3564-0.0392 i$.

Since all Eigen values are negative (no sign change in the first column of the Routh-Herwitz table), then the system is stable which implies $R_{c 0}<1$; then, Khat chewing-free equilibrium point $E_{c *}^{*}$ is stable. Hence, Khat chewing-free equilibrium point $E_{c *}$ is also stable. Therefore, the number of Khat chewers is reduced to zero.

For column 4,

$$
\begin{aligned}
J(n, e, c, \chi) & =\left(\begin{array}{cccc}
-\epsilon & -\rho & 0 & 0 \\
0 & \rho-\epsilon-\alpha_{1} & \alpha_{2} & 0 \\
0 & \alpha_{1} & -\epsilon-\alpha_{3}-\nu-\alpha_{2} & \alpha_{4} \\
0 & 0 & \alpha_{3} \omega & -\alpha_{4}-\delta-\epsilon
\end{array}\right), \\
J(1,0,0,0) & =\left(\begin{array}{cccc}
-0.3234 & -0.9 & 0 & 0 \\
0 & -0.1 & 0.65 & 0 \\
0 & 0.68 & -1.77 & 0.79 \\
0 & 0 & 0.16 & -1.7
\end{array}\right)
\end{aligned}
$$

Its characteristic equation is $\theta^{4}+3.89 \theta^{3}+$ $3.94 \theta^{2}+0.44 v-0.15=0$, and its roots are -2.2140 , $-1.4918,-0.3242$, and 0.1401 . There is a sign change in the first column of the Routh-Herwitz table or the roots have no same signs; then, the system is unstable which implies $R_{c 0}>1$; then, Khat chewing-free equilibrium point $E_{c *}^{*}$ is unstable. Hence, Khat chewing-free equilibrium point $E_{c *}$ is also unstable. $E_{c *}$ is unstable or $R_{c 0}>1$, which indicates an insignificant inflow of Khat chewers into the community will produce a large number of Khat chewers.

4.3. Stability Analysis of Khat Chewing-Present Equilibrium Point $E_{c *}^{p *}$. We analyzed the stability of Khat chewing- present equilibrium point $E_{c *}^{p *}=(-286,212,200,18.5)$, using estimated values of column 2 of Table 2 as follows.

$J\left(n_{1}, e_{1}, c_{1}, \chi_{1}\right)=$
$\left(\begin{array}{cccc}-\epsilon-\rho e_{1} & -\rho n_{1} & 0 & 0 \\ \rho e_{1} & \rho n_{1}-\epsilon-\alpha_{1} & \alpha_{2} & 0 \\ 0 & \alpha_{1} & -\epsilon-\alpha_{3}-\nu-\alpha_{2} & \alpha_{4} \\ 0 & 0 & \alpha_{3} \omega & -\alpha_{4}-\delta-\epsilon\end{array}\right)$,

where

$$
J(-286,212,200,18.5)=\left(\begin{array}{cccc}
-0.4 & 0.11 & 0 & 0 \\
0.08 & .-0.5 & 0.0085 & 0 \\
0 & 0.07 & -1.13 & 0.79 \\
0 & 0 & 0.16 & -1.7
\end{array}\right)
$$

Its characteristics equation is $\theta^{4}+3.78 \theta^{3}+4.53 \theta^{2}+$ $2.15 \theta+0.34=0$ :

$$
-2.03,-0.71+0.06 i,-0.71-0.06 i \text {, and }-0.33 \text {. }
$$

There is no sign change in the first column of the Routh-Herwitz table or the roots have the same sign; then, the system is stable; this implies that $R_{c 0}<1$, and $E_{c *}^{p *}$ is unstable. Hence, $E_{c *}^{p}$ is unstable.

4.4. Numerical Simulation. In this section, we cherry-pick which parameter affects the model in the event of an uncontrolled spread of Khat chewers in the community and which parameter affects the model when the number of Khat chewers in the community decreases. For initial conditions $n_{0}=1000, e_{0}=800, c_{0}=600, \chi_{0}=400$, and $h_{0}=300$ and using column 1 of Table 2, we obtained Figure 2 which illustrates that the number of non-Khat chewers, the number of exposed, number of Khat chewers, and the number of 


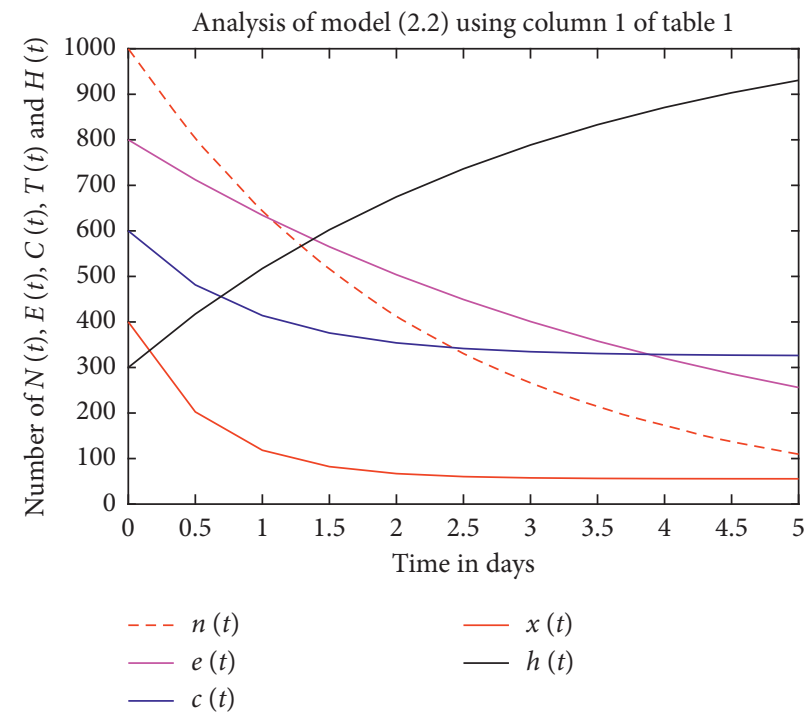

Figure 2: Phase portrait of our model for $R_{c 0}=0.39$ and $R_{c 0}\left(E_{c *}\right)=0.00039$, and $E_{c *}$ is stable.

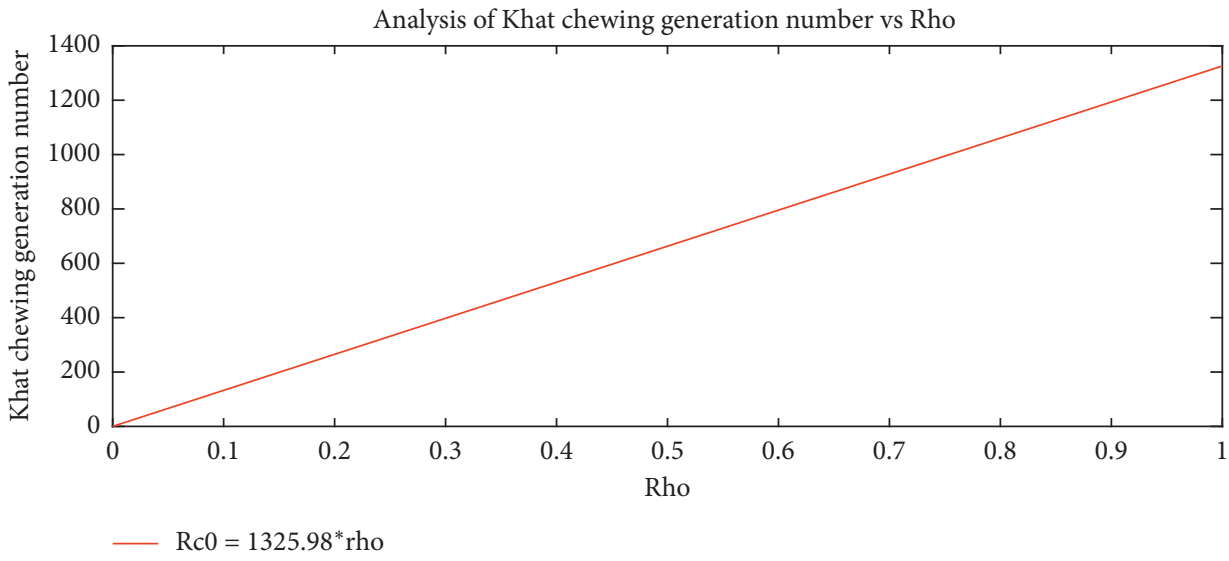

FIgURE 3: The Khat-chewing generation number increases as the value of $\rho$ increases.

temporary Khat chewers reduced or approaches to zero as time rises, and the number of permanent Khat chewers increases as time rises.

For initial conditions $n_{0}=1000, e_{0}=800, c_{0}=600$, $\chi_{0}=400$, and $h_{0}=300$ and using column 1 of Table 2 , we obtained Figure 3 which demonstrates that the Khatchewing generation number increases as the value of $\rho$ increases. Hence, if $\rho<0.00075, R_{c 0}<1$, and the number of Khat chewers reduces to zero, and if $\rho>0.00075, R_{c 0}>1$, andthe inflow of an insignificant number of Khat chewers into the community produces a significant number of Khat chewers.

For initial conditions $n_{0}=1000, e_{0}=800, c_{0}=600$, $\chi_{0}=400$, and $h_{0}=300$ and using column 1 of Table 2, we obtained Figure 4 which demonstrates that the Khatchewing generation number decreases as the value of $\alpha_{2}$ increases; then, the spreading of Khat chewers declines as $\alpha_{2}$ increases.
For initial conditions $n_{0}=1000, e_{0}=800, c_{0}=600$, $\chi_{0}=400$, and $h_{0}=300$ and using column 4 of Table 2, we obtained Figure 5 which represents that the Khat-chewing generation number increases as the value of $\rho$ increases. Therefore, for $\rho>0, R_{c 0}>1$, andthe inflow of an insignificant number of Khat chewers into the community produces a significant number of Khat chewers.

For initial conditions $n_{0}=1000, e_{0}=800, c_{0}=600$, $\chi_{0}=400$, and $h_{0}=300$ and using column 4 of Table 2, we obtained Figure 6 which symbolizes that the Khat-chewing generation number increases as the value of $\alpha_{2}$ increases in column 4 of Table 2 for $0 \leq \alpha_{2} \leq 1$. From this graph we conclude that the inflow of a insignificant number of Khat chewers into the community produces a significant number of Khat chewers even the rate of return back from Khat chewing to exposed because of socio-economic, environmental, and religious factors increases. Thus, to turn down the spreading of Khat chewers to the community, the rate of 


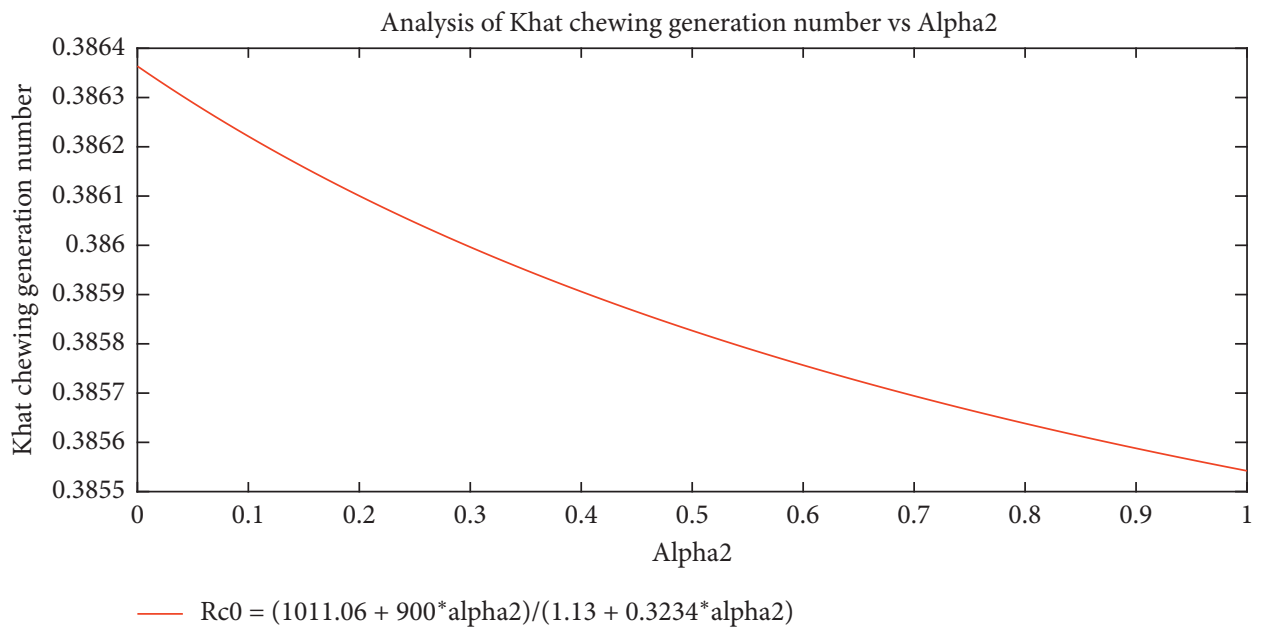

FIgURE 4: Khat-chewing generation number decreases as the value of $\alpha_{2}$ increases.

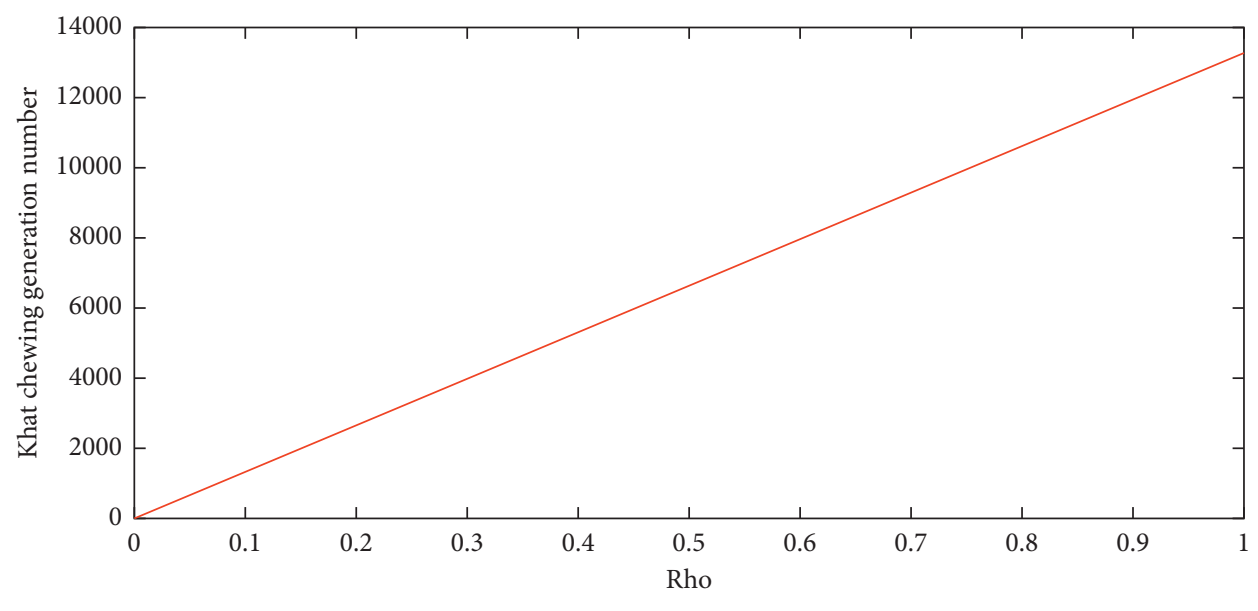

$\mathrm{Rc} 0=13263.7^{*}$ rho

FIGURE 5: Khat-chewing generation number increases as the value of $\rho$ increases.

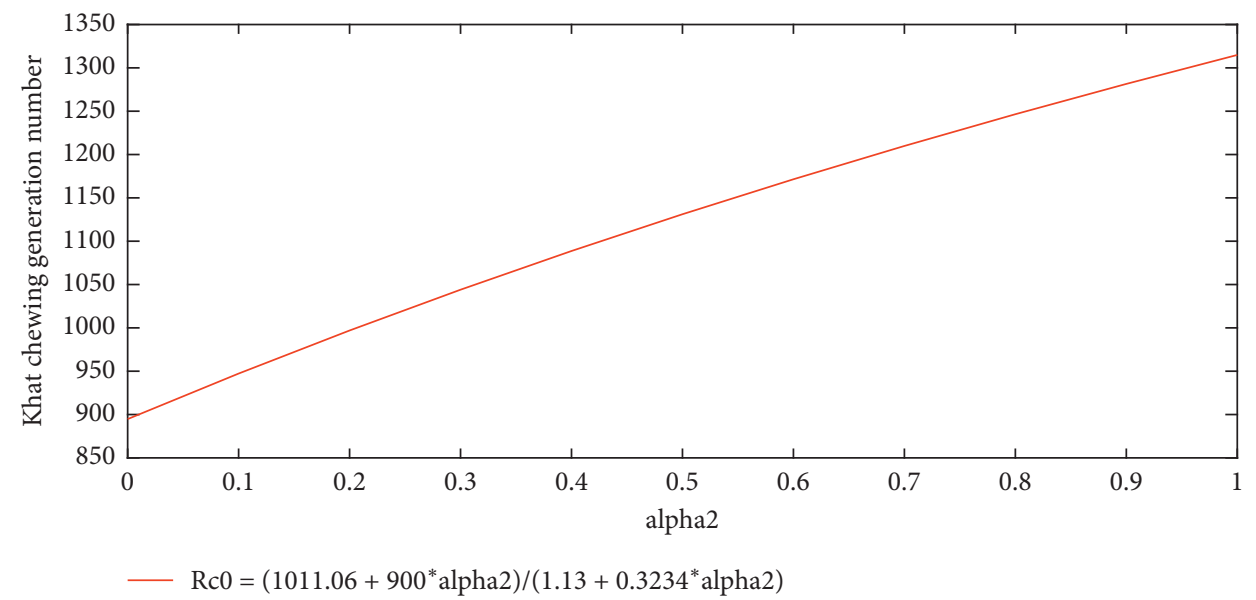

FIGURE 6: The Khat-chewing generation number increases as the value of $\alpha_{2}$ increases. 
return from Khat chewing to an exposed group because of socio-economic, environmental and religious influences must grow exponentially.

\section{Conclusion and Recommendation}

In this study, we examined the return from Khat chewing to the exposed group due to socio-economic, environmental, and religious factors, as well as the rate of conversion from Khat chewing to the exposed group of consequences in the Khat chewing dynamics' model. We have derived the Khat chewing generations' number $R_{c 0}$ and Khat chewing-free and Khat chewing-present equilibrium points. For $R_{c 0}<1$, the Khat chewing-free equilibrium point is stable, and it is unstable for $R_{c 0}>1$, and the Khat chewing-present equilibrium point is stable if $R_{c 0}>1$, and it is unstable if $R_{c 0}<1$.

The stability of the two equilibrium points implies that the influx of the insignificant number of Khat chewers into the community produces a significant number of Khat chewers if the conversion rate of nonchewers to vulnerable groups is high, and the inflow of Khat chewers to the community produces an insignificant number of Khat chewers if they return back from Khat chewing to the exposed group because socio-economic, environmental, and religious influences raise rapidly. We recommend to the community to stop or minimize chewing Khat habits because the practice of chewing Khat leads to economic crises, social crises (turn into isolated), and health crises, and since media awareness are a very effective tool to generate precautionary control measures of communicable diseases [22], so both governmental and nongovernmental health office stakeholders must alert their people about the impact of Khat chewing through media. In addition, to future researchers, we recommend them to extend the model by grouping the entire population based on sex, religion, and geographic locations.

\section{Data Availability}

The data used to support the findings of this study are included within the article.

\section{Conflicts of Interest}

The authors declare that they have no conflicts of interest regarding the publication of this paper.

\section{References}

[1] N. Numan, “The green leaf: khat," World Journal of Medical Sciences, vol. 4, pp. 210-223, 2012.

[2] A. Alem, D. Kebede, and G. Kullgren, "The prevalence and socio-demographic correlates of khat chewing in Butajira, Ethiopia," Acta Psychiatrica Scandinavica, vol. 100, pp. 84-91, 1999.

[3] E. Gebissa, "Scourge of life or an economic lifeline? public discourses on khat (Catha edulis) in Ethiopia," Substance Use \& Misuse, vol. 43, no. 6, pp. 784-802, 2008.

[4] I. C. Eschart, P. E. Parker, W. J. Weidner, J. M. Dabney, J. B. Scott, and F. J. Haddy, "Coronary vascular and myocardial responses to carotid body stimulation in the dog," The American Journal of Physiology, vol. 229, no. 3, p. 754, 1975.

[5] M. George, K. Stephen, and D. Theuri, "Modelling Miraa addiction like a disease incorporating voluntary quitting," Journal of Advances in Mathematics and Computer Science, vol. 24, no. 4, pp. 1-19, 2017.

[6] A. Gashaw and T. Getachew, "The chemistry of khat and adverse effect of khat chewing," American Scientific Research Journal for Engineering, Technology, and Sciences, vol. 9, no. 1, pp. 35-46, 2014.

[7] A. Schmoldt, H. F. Benthe, and G. Haberland, "Digitoxin metabolism by rat liver microsomes," Biochemical Pharmacology, vol. 24, no. 17, p. 1639, 1975.

[8] H. Degol, Should Khat Should be Banned? the Development Impact, International Poverty Centre, Caribbean, West Indian, 2007.

[9] L. S. Kandari, H. R. Yadav, A. K. Thakur, and T. Kandari, "Chat (Catha edulis): a socio economic crop in Harar region, Eastern Ethiopia," SpringerPlus, vol. 3, p. 579, 2014.

[10] B. W. Ademe, C. C. Jennifer, A. Dalsgaard, and L. Brimer, "Khat production and consumption; its implication on land area used for crop production and crop variety production among rural household of Ethiopia," Journal of Food Security, vol. 5 , no. 4 , p. $148,2017$.

[11] L. Cochrane and D. O’Regan, "Legal harvest and illegal trade: trends, challenges, and options in khat production in Ethiopia," International Journal of Drug Policy, vol. 30, pp. 27-34, 2016.

[12] D. Haile and Y. Lakew, "Khat chewing practice and associated factors among adults in Ethiopia: further analysis using the 2011 demographic and health survey," PLoS One, vol. 10, no. 6, 2015.

[13] A. Yahya, Y. Rajeshwar, T. Eticha et al., "Socio-economic and health effects of khat chewing in Mekelle, Tigray Region, Ethiopia," International Journal of Pharmacy and Pharmaceutical Research, vol. 8, p. 13, 2016.

[14] A. Adugna, T. Azale, and S. Handebo, 2020 Intention to stop khat chewing and associated factors among khat chewers in Gondar City, Northwest Ethiopia: using trans theoretical model.

[15] P. E. Alele and J. B. Rujumba, "Khat (Catha edulis) and ethanol co-dependence modulate seizure expression in a pentylenetetrazol seizure model," Journal of Ethnopharmacology, vol. 137, no. 3, pp. 1431-1436, 2011.

[16] M. D. N. Abid, J. Chen, M. Xiang, J. Zhou, X. Chen, and F. Gong, "Khat (Catha edulis) generates reactive oxygen species and promotes hepatic cell apoptosis via MAPK activation," International Journal of Molecular Medicine, vol. 32, no. 2, pp. 389-395, 2013.

[17] J. Järvisalo and N. Saris, "Action of propranolol on mitochondrial functions--effects on energized ion fluxes in the presence of valinomycin," Biochemical Pharmacology, vol. 24, no. 18, p. 1701, 1975.

[18] R. Krizevski, N. Dudai, E. Bar, and E. Lewinsohn, "Developmental patterns of phenylpropylamino alkaloids accumulation in khat (Catha edulis, Forsk.)," Journal of Ethnopharmacology, vol. 114, no. 3, 2007.

[19] P. Griffiths, D. Lopez, R. Sedefov et al., "Khat use and monitoring drug use in Europe: the current situation and issues for the future," Journal of Ethnopharmacology, vol. 132, no. 3, 2010. 
[20] S. A. Koshin, "Khat and COVID-19 Somalia's cross-border economy in the time of coronavirus," Rift Valley Institute Briefing Paper, pp. 1-4, 2020.

[21] R. Belwal and H. Teshome, "Chat exports and the Ethiopian economy: opportunities, dilemmas and constraints," African Journal of Business Management, vol. 5, no. 9, p. 3635, 2011.

[22] D. K. Das, S. Khajanchi, and T. K. Kar, "The impact of the media awareness and optimal strategy on the prevalence of tuberculosis," Applied Mathematics and Computation, vol. 366, Article ID 124732, 2020.

[23] S. Khajanchi, D. K. Das, and T. K. Kar, "Dynamics of tuberculosis transmission with exogenous reinfections and endogenous reactivation," Physica A: Statistical Mechanics and its Applications, vol. 497, pp. 52-71, 2018.

[24] D. K. Das, S. Khajanchi, and T. K. Kar, "Transmission dynamics of tuberculosis with multiple re-infections," Chaos, Solitons \& Fractals, vol. 130, Article ID 109450, 2020.

[25] D. K. Das, S. Khajanchi, and T. K. Kar, "Influence of multiple re-infections in tuberculosis transmission dynamics: a mathematical approach," in Proceedings of the 2019 8th International Conference on Modeling Simulation and Applied Optimization (ICMSAO), Manama, Bahrain, April 2019.

[26] P. Samui, J. Mondal, and S. Khajanchi, "A mathematical model for COVID-19 transmission dynamics with a case study of India," Chaos, Solitons \& Fractals, vol. 140, 2020.

[27] R. M. Anderson and R. M. May, "Population biology of infectious diseases: part I," Nature, vol. 280, no. 5721, pp. 361-367, 1979.

[28] R. P. Durbin and T. T. Mekonnen, "Letter: acid secretion by gastric mucous membrane," The American Journal of Physiology, vol. 229, no. 6, p. 1726, 1975.

[29] K. Sarkar, S. Khajanchi, and J. J. Nieto, "Modeling and forecasting the COVID-19 pandemic in India," Chaos, Solitons \& Fractals, vol. 139, p. 110049, 2020.

[30] S. Khajanchi, S. Bera, and T. K. Roy, "Mathematical analysis of the global dynamics of a HTLV-I infection model, considering the role of cytotoxic T-lymphocytes," Mathematics and Computers in Simulation, vol. 180, pp. 354-378, 2021.

[31] O. Diekmann, J. A. P. Heesterbeek, and J. A. J. Metz, "On the definition and the computation of the basic reproduction ratio $\mathrm{R} 0$ in models for infectious diseases in heterogeneous populations," Journal of Mathematical Biology, vol. 28, no. 4, 1990.

[32] K. Dietz, "The estimation of the basic reproduction number for infectious diseases," Statistical Methods in Medical Research, vol. 2, no. 1, pp. 23-41, 1993.

[33] P. Van Den Driessche and J. Watmough, "Reproduction numbers and sub-threshold endemic equilibria for compartmental models of disease transmission," Mathematical Biosciences, vol. 180, no. 1-2, pp. 29-48, 2002.

[34] I. Hussain and R. Herwitz, http://imtiazhussainkalwar.weebly. comStability criterion." lecture 19-20, 2020.

[35] J. W. McEvoy, M. J. Blaha, J. J. Rivera et al., "Mortality rates in smokers and nonsmokers in the presence or absence of coronary artery calcification," JACC: Cardiovascular Imaging, vol. 5, no. 10, pp. 1037-1045, 2012. 